\title{
A Review on Edge Detection Using Different Techniques
}

\author{
Rekha Rani $^{1}$, Shailja Kumari ${ }^{2}$ \\ ${ }^{1}$ Research Fellow (M. Tech), Department of Computer Science, Ch. DeviLal University Sirsa, Haryana \\ ${ }^{2}$ Assistant Professor, Department of Computer Science, Ch. DeviLal University Sirsa, Haryana
}

\begin{abstract}
An edge can be described as the boundary between an object and the background in an image, and it also indicates the boundary between overlapping objects in an image. Edge detection methods are a combination of image smoothing and image differentiation plus a post-processing for edge labelling. Edge detection discusses the process of identifying and locating sharp discontinuities in an image. In this paper, the main aim is to survey the theory of edge detection for image processing using different types of techniques. In this paper, the represented methods for edge segmentation of satellite images; that used five techniques for this category; Sobel operator technique, Roberts Cross Edge Detector, Prewitt technique, Laplacian technique and Edge Maximization Technique (EMT). In the first section of the paper describe the Introduction of edge detection. Second section of the paper described the Edge Detection Techniques. In the third section describe analysis the Related Work. In the fourth section we described different steps of Fuzzy Logic Using Logic Using Edge Detection. In the fifth section described CBIR Technique and Bacterial Foraging Optimization Technique. Finally presented the Conclusion with the references.
\end{abstract}

Keywords: Edge detection, Fuzzy Logic, CBIR Technique, Bacterial Foraging Optimization Technique.

\section{Introduction}

Edge detection is more common for detecting discontinuities in gray level than detecting isolated points and thin lines because isolated points and thin lines so not occur frequently in most practical images. Edge is a boundary between two homogeneous regions. Edge detection refers to the process of identifying and locating sharp discontinuities in an image. Edge detection is an important pre-processing step for any image processing application, object recognition and object detection. Edge detection is very helpful in case of noise free images. But in case of noisy images it is a challenging task. Noisy images are corrupted images. Their parameters are difficult to analyze and detect.Edge detection is a fundamental tool used in most image processing applications to obtain information from the frames as a precursor step to feature extraction and object segmentation. This process detects outlines of an object and boundaries between objects and the background in the image. An edge-detection filter can also be used to improve the appearance of blurred image.

The most common edge types are steps, lines and junctions. The step edges are mainly produced by a physical edge, an object hiding another or a shadow on a surface. It generally occurs between two regions having almost constant, but different, grey levels. The step edges are the points at which the grey level discontinuity occurs, and localized at the inflection points. They can be detected by using the gradient of intensity function of the image. Step edges are localized as positive maxima or negative minima of the first-order derivative or as zero-crossings of the second-order derivative (Figure 1).

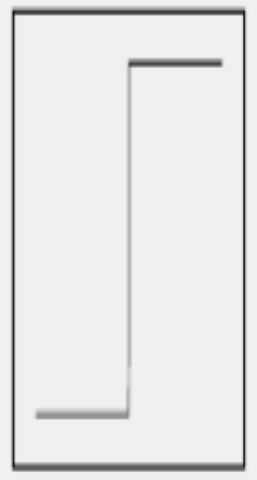

d.

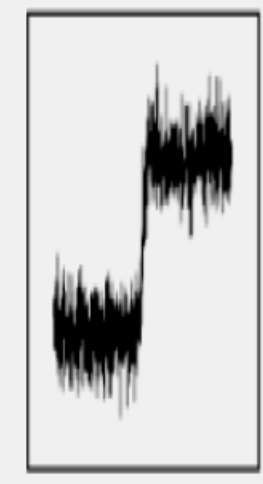

b.
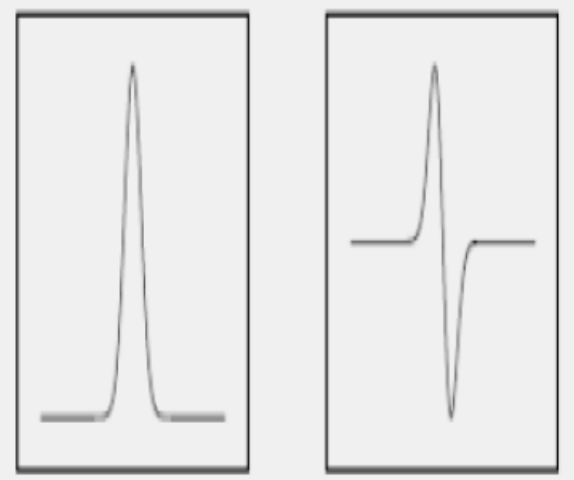

C.

Figure 1: Profile of (a) ideal step edge (b) smoothed step edge corrupted by noise (c) first-order derivative (d) second-order derivative of the smoothed step edge corrupted by noise

\section{Edge Detection Techniques}

A. Edge Detection:- Canny finds edges by looking for local maxima of the gradient of $f(x, y)$. The gradient is calculated using the derivative of a Gaussian filter. The method uses two thresholds to detect strong and weak edges, and includes the

Volume 5 Issue 6, June 2016 www.ijsr.net 


\section{International Journal of Science and Research (IJSR) \\ ISSN (Online): 2319-7064}

Index Copernicus Value (2013): 6.14 | Impact Factor (2015): 6.391

weak edges in the output only if they are connected to strong edges. Therefore, this method is more likely to detect true weak edges. The implemented Canny edge detector presented the best performance Both visually and quantitatively based on the measures such as mean square distance, error edge map and signal to noise ratio. Using the implemented canny edge detector as an enhancement tool for remote sensing images, the result was robust and achieved a very high enhancement level.

I. Sobel Operators:- Sobel Operators are the computation of the partial derivation in gradient may be approximated in digital images. This technique performs 2D spatial gradient measurement on an image and also it emphasizes regions of high spatial frequency that correspond to edges. Typically it is used to find the approximate absolute gradient magnitude at each point in an input grayscale image. In theory at least, the operator consists of a pair of $3 \times 3$ convolution masks as shown in figure. One mask is simply the other rotated by $90^{\circ}$. These masks can be separate measurements of the gradient component in each orientation that is Gx and Gy. These can be combined together to find the absolute magnitude of the gradient at each point and the orientation of that gradient. The convolution masks of the Sobel detector are given below-

\begin{tabular}{|c|c|c|}
\hline-1 & 0 & 1 \\
\hline-2 & 0 & 2 \\
\hline-1 & 0 & 1 \\
\hline 1 & 2 & 1 \\
\hline 0 & 0 & 0 \\
\hline-1 & -2 & 1 \\
\hline
\end{tabular}

Figure I: The Sobel masks

These two masks together with any of the equations:

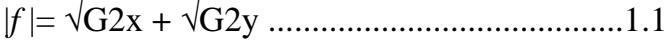

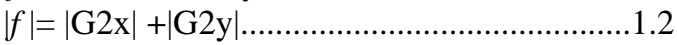

II. Roberts Cross Edge Detector: The Roberts Cross operator performs a simple, quick to compute, 2-D spatial gradient measurement on an image. It thus highlights regions of high spatial frequency which often correspond to edges. In its most common usage, the input to the operator is a grayscale image, as is the output. Pixel values at each point in the output represent the estimated absolute magnitude of the spatial gradient of the input image at that point. The convolution masks of the Roberts detector are given below-

\begin{tabular}{|c|c|}
\hline 1 & 0 \\
\hline 0 & -1 \\
\hline 0 & +1 \\
\hline-1 & -0 \\
\hline
\end{tabular}

Figure II: Roberts cross convolution mask

III. Prewitt Operator:- The prewitt operator is an approximate way to estimate the magnitude and orientation of the edge. The prewitt operator uses the same equations as the Sobel operator, except the constant $\mathrm{k}=1$ by Comparing to Sobel operator. This prewitt operator does not place any emphasis on pixels that are closer to the center of the masks.This operator will measure two components. The vertical edge component is calculated with kernel Gx and the horizontal edge component is calculated with kernel Gy. $|\mathrm{Gx}|$
$+|\mathrm{Gy}|$ give an indication of the intensity of the gradient in the current pixel. The convolution masks of the Prewitt detector are given below-

\begin{tabular}{|c|c|c|}
\hline-1 & 0 & 1 \\
\hline-1 & 0 & 1 \\
\hline-1 & 0 & 1 \\
\hline 1 & 1 & 1 \\
\hline 0 & 0 & 0 \\
\hline-1 & -1 & -1 \\
\hline
\end{tabular}

Figure III: Prewitt mask

IV. Laplacian Operator:- The Laplacian of an image $f(x, y)$ is a second order Derivative defined as:

$\Delta^{2} f=\partial^{2} f / \partial x^{2}+\partial^{2} f / \partial y^{2}$.

The Laplacian is usually used to establish whether a pixel is on the dark or light side of an edge. The convolution masks of the Laplacian detector are given below-

\begin{tabular}{|c|c|c|}
\hline 0 & -1 & 0 \\
\hline-1 & 4 & -1 \\
\hline 0 & -1 & 0 \\
\hline
\end{tabular}

Figure IV: The Laplacian masks

V. Edge Maximization Technique (EMT):- In images when there is more than one homogeneous region e.g. an image has many objects with different gray levels or where there is a change on illumination between the objects and its background. In this case, portion of the objects may be merged with the background or portions of the background may appear as an object.

B. Complement Image:- This process is to transform an edge image into regions as well as reduce unnecessary noise such as spurs to provide a clean region image. Applying binary invert operator to the binary edge image gives a region image in binary with 1's where a region found a 0 's elsewhere is given.

In the complement of a binary image, black becomes white and white becomes black. This operation facilitates further morphological filtering and smoothing operations which are carried out in next phases for fine edge detection.

C. Morphological Open operator:- Morphology relates to structure or form of objects. Morphological filtering simplified segmented images by smoothing out object outlines filling small holes, eliminating small projections. Primary operations are dilation and erosion. These operations use a structuring element which determines exactly how object will be dilated or eroded. Dilation process expands image objects by changing pixels with value of " 0 " to " 1 ". On the other hand the erosion process shrinks binary objects by changing pixels with a value of " 1 " to " 0 ". There is also a combination of dilation and erosion called opening and closing. Opening is erosion followed by dilation

D. Morphological Erosion:- The morphological erosion operator is applied for localization of edges image that shrinks region-pixels in size which make clear image. Figure shows the comparison of the edge detection's for the example image.

\section{Volume 5 Issue 6, June 2016} www.ijsr.net 


\section{International Journal of Science and Research (IJSR) \\ ISSN (Online): 2319-7064}

Index Copernicus Value (2013): 6.14 | Impact Factor (2015): 6.391

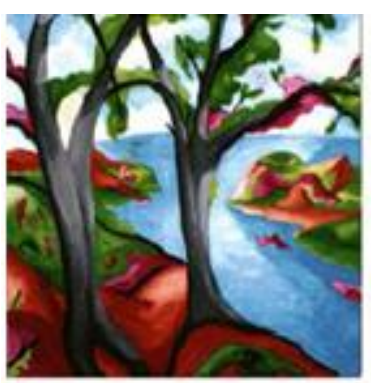

(a)

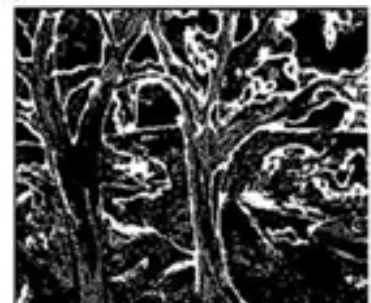

(e)

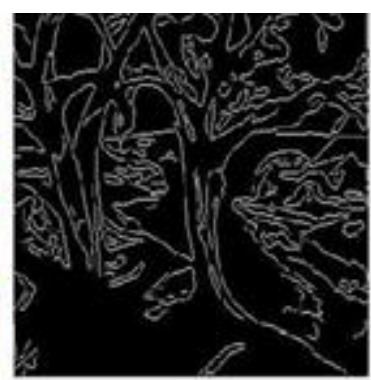

(b)

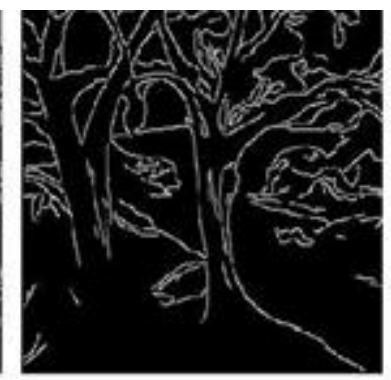

(c)

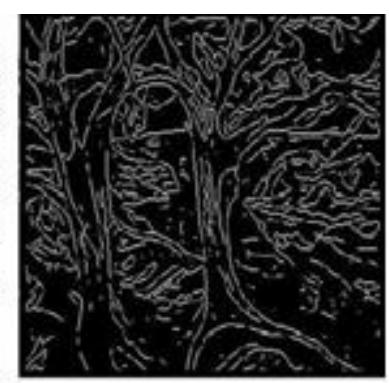

(d)

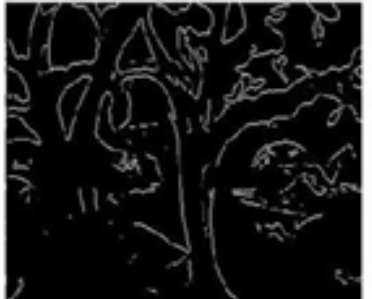

(f)

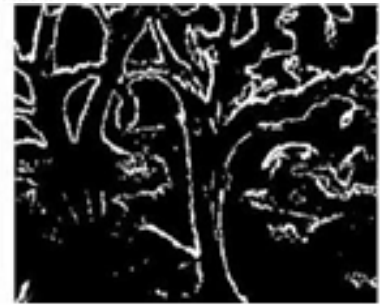

(g)

Figure V: (a) Original Trees image, (b) Sobel Edge Detector (c) Roberts Cross Edge Detector (d) Prewitt Edge Detector (e) Laplacian Edge Detector (f) Edge Maximization Technique Edge Detector and (g) The proposed approach

\section{Literature Survey}

3.1 Shihu Zhu et. Al. proposed the new method of edge detection based on multi-structure elements morphology and image fusion. Edges are detected using four different orientations SE (structure element) where direction angles of all the structure elements are $0 \square, 45 \square, 90 \square, 135 \square$ and final edge result is got by image fusion using entropy weighted method. The proposed method not only can effectively eliminate the image noise, but also effectively maintain good edge information.

3.2 C.Naga Rajuet. Al. propose dan edge detection algorithm based on multi-structure elements morphology. The eight different edge detection results are obtained by using morphology gradient algorithm and final edge results are obtained by using synthetic weighted method. The proposed algorithm results are compared with the conventional mathematical morphological edge detection and differential edge detection operators such as Watershed method, Sobel operator and Canny operator and obtained the better edges over traditional methods.

3.3 Wenshuo Gao e. al.proposed a method which combines Sobel edge detection operator and soft-threshold wavelet de-noising for edge detection. This method used on images which include White Gaussian noises. The widely used operators such as Sobel, Prewitt, Roberts and Laplaca in are sensitive to noises and their anti-noise performances are poor. This paper proposes an edge detection method which combines soft-threshold wavelet de-noising and Sobel Operator, its anti-noise performance is very strong. Firstly soft-threshold wavelet used to remove noise, then Sobel edge detection used for edge detection on the image. The effect by using this method to do edge detection is very good and can remove the noise effectively.

3.4 Sabina Priya darshiniet. Al.proposed a new technique of edge detection that employs simple additions and divisions and finds out fine edges. It makes use of a threshold that is computed automatically during the edge detection process and it's simple to compute the threshold value. It is based upon simple arithmetic and logic operations, consisting of three procedures: image binarizati on, image contraction and image subtraction. The proposed method is a computationally simpler and performs better than Sobel's method and require much lesser computation than Sobel's method.

3.5 M Rama Bai , Dr V Venkata Krishna described the new morphological approach for noise removal cum edge detection for both binary and gray scale images.

3.6Tzu-Heng Henry Lee and Taipei, Taiwan, ROC described the various edge detection algorithms and detector design methods.

3.7 Mitra Basu, Senior Member presented a survey of Gaussian-based edge detection techniques. This described in a gray level image of an edge. Edge detection is the process which detects the presence and locations of these intensity transitions.

3.8 Mohamed A. El-Sayed described the hybrid entropic edge detector and proposed a method to decrease the computation time and generate high quality of edge detection

3.9 Peter Wilkins, Paul Ferguson, Alan F. Smeaton and Cathal Gurrin described the approach to reduce the search space for image retrieval. A fair degree of overlap can be achieved in a reduced subset that can be retrieved in a timely manner .

3.10 M. Sujaritha and S. Annadurai described a novel level set method for color image segmentation which using Binary Level-set Partitioning Approach . It eliminates the need of the re-initialization, calculation of number of regions procedure which is very costly.

\section{Fuzzy Logic Using Edge Detection}

A fuzzy logic reasoning strategy is proposed for edge detection in digital images without determining the threshold 


\section{International Journal of Science and Research (IJSR) \\ ISSN (Online): 2319-7064}

Index Copernicus Value (2013): 6.14 | Impact Factor (2015): 6.391

value or need training algorithm.

\section{Fuzzy Image Processing}

Fuzzy image processing is the collection of all approaches that understand, represent and process the images, their segments and features as fuzzy sets. The representation and processing depend on the selected fuzzy technique and on the problem to be solved. Fuzzy image processing has three main stages: image fuzzification, modification of membership values and image defuzzification.

\section{Fuzzy Sets and Fuzzy Membership Functions}

The system implementation was carried out considering that the input image and the output image obtained after defuzzification are both 8-bit quantized; this way, their gray levels are always between 0 and 255. The fuzzy sets were created to represent each variable's intensities; these sets were associated to the linguistic variables "Black", "White" and "Gray" of an edge and The functions adopted to implement the "and" and "or" operations were the minimum and maximum functions, respectively. The values of the membership's function of the output are designed to separate the values of the blacks, whites and edges of the image.

\section{Inference Rules Definitions}

The inference rules is depends on the weights of the eight neighbors gray level pixels, if the neighbors weights are degree of blacks or degree of whites. The power of these rules is the ability of extract all edges in the processed image directly. This study is as saying all the pixels of the processed image by studying the situation of each neighbor of each pixel.

\section{CBIR Technique and Bacterial foraging optimization Technique}

\subsection{CBIR Technique}

To retrieve the images from the image data there are two techniques, text based and content based image retrieval techniques (CBIR). Content Based Image Retrieval (CBIR) algorithms are means to access a digital image from a database. In CBIR the actual contents of the image are used to describe and analyze an image. The contents of an image are used to form a set of feature vectors using the method involved. Various techniques are used for extraction of the feature vectors for an image. Some of these techniques involve using attributes like shape, color, textures and edge density of an image to extract the feature vector.

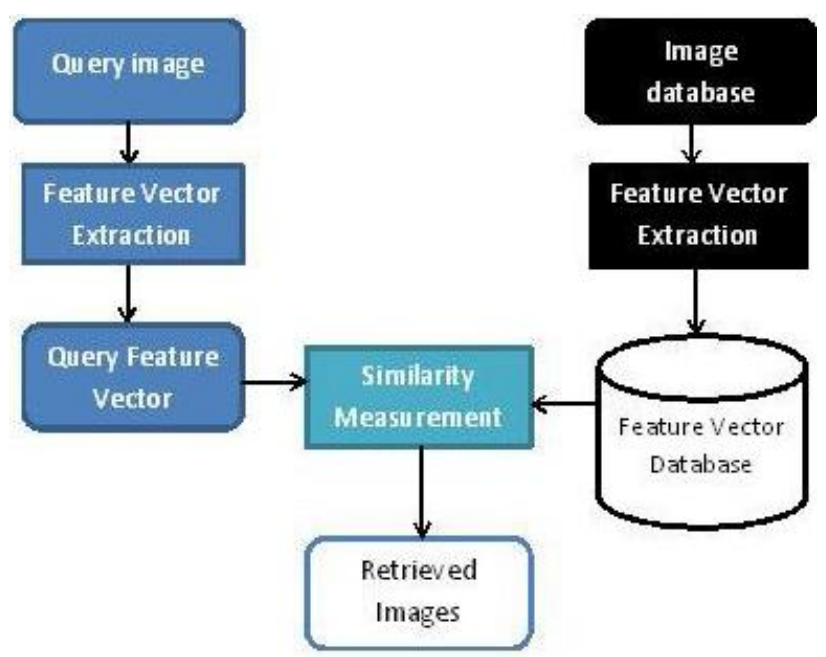

Figure 6: general model for a CBIR system

\subsection{Bacterial foraging optimization Technique}

A new evolutionary technique, called bacterial foraging scheme appeared by Liu and Passino (2002). Foraging can be modeled as an optimization process where bacteria seek to maximize the energy obtained per unit time spent during foraging. A set of bacteria tries to reach an optimum cost by following four stages: Chemo taxis, swarming, reproduction, and elimination and dispersal. To start with, there will be as many solutions as the number of bacteria. Each bacterium produces a solution iteratively for a set of optimal values of parameters. The movement stage of bacteria accomplished through swimming and tumbling. Furthermore, in the reproduction the least healthy bacteria die and of the healthiest, each bacterium splits into two bacteria, which are placed at the same location. While in the elimination and dispersal stage, any bacterium from the total set can be either eliminated or dispersed to a random location during the optimization. Generally the bacteria move for a longer distance in a friendly environment. BFOA mimics the four principal mechanisms observed in a real bacterial system.Chemotaxis, swarming, reproduction, and elimination-dispersal to solve this non-gradient optimization problem. A virtual bacterium is actually one trial solution that moves on the functional surface to locate the global optimum. i) Chemotaxis: This process simulates the movement of an E.coli cell through swimming and tumbling via flagella. Biologically an E.coli bacterium can move in two different ways. It can swim for a period of time in the same direction or it may tumble, and alternate between these two modes of operation for the entire lifetime.

ii) Swarming: An interesting group behavior has been observed for several motile species of bacteria including E.coli and S. typhimurium, where intricate and stable spatio-temporal patterns (swarms) are formed in semisolid nutrient medium. A group of E.coli cells arrange themselves in a traveling ring by moving up the nutrient gradient when placed amidst a semisolid matrix with a single nutrient chemo-effecter. The cells when stimulated by a high level of succinate, release an attractant aspertate, which helps them to aggregate into groups and thus move as concentric patterns of swarms with high bacterial density.

iii) Elimination and Dispersal: Gradual or sudden changes in the local environment where a bacterium population lives may occur due to various reasons e.g. a significant local rise 


\section{International Journal of Science and Research (IJSR) \\ ISSN (Online): 2319-7064}

Index Copernicus Value (2013): 6.14 | Impact Factor (2015): 6.391

of temperature may kill a group of bacteria that are currently in a region with a high concentration of nutrient gradients. Events can take place in such a fashion that all the bacteria in a region are killed or a group is dispersed into a new location. To simulate this phenomenon in BFOA some bacteria are liquidated at random with a very small probability while the new replacements are randomly initialized over the search space.

\section{Conclusion}

In this paper general edge detection techniques are discussed to check out how an image is fetched out from the database. The purpose of the work is to retrieve images with less noise. Images with noise in the database can be reduced with different techniques including the CBIR method. To design an algorithm to work on the parameters of the corrupted noisy images, BFOA has been devised as a form of preferential treatment because BFOA check out all pixels connected to edge pixels so that the problem of edge detection due to noise can be mitigated.The Bacterial Foraging Optimization Algorithm belongs to the field of Bacteria Optimization Algorithms and Swarm Optimization, and more broadly to the fields of Computational Intelligence and Metaheuristics. It is related to other Bacteria Optimization Algorithms such as the Bacteria Chemotaxis Algorithm and other Swarm Intelligence algorithms such as Ant Colony Optimization and Particle Swarm Optimization.

\section{References}

[1] Tzu-Heng Henry Lee and Taipei, Taiwan ROC, "Edge Detection Analysis", IJCSI International Journal of Computer Science Issues, Vol. 5, Issue 6, No 1, September 2012.

[2] AkanshaMehrotra,KrishnaKantSingh,M.J.Nigam, "A Novel Algorithm for Impulse Noise Removal and Edge Detection", International Journal of Computer Applications, Vol. 38,No.7,January 2012.

[3] Mohamed A. El-Sayed, "A New Algorithm Based Entropic Threshold for Edge Detection in Images", IJCSI International Journal of Computer Science Issues, Vol. 8, Issue 5, No 1, September 2011.

[4] Khang Siang Tan, Nor Ashidi Mat Isa, “Color image segmentation using histogram thresholding" Fuzzy Cmeans hybrid approach, K. Siang Tan, N.A. Mat Isa / Pattern Recognition 44 ( 1-15) (2011)

[5] Dr. H. B. Kekre, Dhirendra Mishra, "Sectorization of Walsh and Walsh Wavelet in CBIR", International Journal on Computer Science and Engineering (IJCSE) Vol. 3 No. 6 June 2011.

[6] Shihu Zhu, "Edge Detection Based on Multi-structure Elements Morphology and Image Fusion", IEEE, 978-1-4244-9600-vol-6, 2011.

[7] C.Naga Raju, S.Naga Mani, G.rakesh Prasad, S.Sunitha, "Morphological Edge Detection Algorithm Based on Multi-Structure Elements of Different Directions", International Journal of Information and Communication Technology Research Volume 1 No. 1, May 2011.

[8] Wenshuo Gao, Lei Yang, Xiaoguang Zhang, Huizhong Liu, "An Improved Sobel Edge Detection", IEEE, 978-1-4244-5540-vol-9,2010.

[9] Mr.Salem Saleh Al-amri,Dr.N.V.Kalyankar,and
Dr.Khamitkar S.D, "Image Segmentation by using edge detection", International Journal of Computer Science and Engineering,Vol.02,No.03,2010.

[10] M Rama Bai, Dr V Venkata Krishna and J SreeDevi , “A new Morphological Approach for Noise Removal cum Edge Detection", IJCSI International Journal of Computer Science Issues, Vol. 7, Issue 6, November 2010.

[11]N. Senthilkumaran, R. Rajesh, "Edge Detection Techniques for Image Segmentation and A Survey of Soft Computing Approaches", International Journal of Recent Trends in Engineering, Vol. 1, No. 2, May 2009.

[12] A.A.Alshennawy, A.A.Aly, "Edge Detection in Digital Images Using Fuzzy Logic Technique", International Journal of Electrical and Engineering ,4:7 2009.

[13] Mitra Basu, Senior Member IEEE, "Gaussian Based Edge-Detection Methods A Survey”, IEEE Transactions on System,man,and cybernetics part c:Application and Reviews, Vol. 32, No. 3, August 2002.

[14]M. Sujaritha and S. Annadurai , "Color Image Segmentation Using Binary Level-set Partitioning Approach",International Journal of Soft Computing, 4: 76-84 (2009).

[15] Jun Zhang, Quieshi Zhang and Jinglu HU, "RGB color Centriods Segmentation(CCS) for face Detection", ICGST-GVIP Journal,ISSN 1687- 398X,Volume (9) ,issue(II),april 2009. 